\title{
Liver resection is beneficial for patients with colorectal liver metastases and extrahepatic disease
}

\author{
Hauke Lang \\ Department of General, Visceral and Transplantation Surgery, University Medical Center, Mainz, Germany \\ Correspondence to: Prof. Dr. Hauke Lang, MA, FACS. Department of General, Visceral and Transplantation Surgery, University Medical Center, \\ Langenbeckstraße 1, 55131 Mainz, Germany. Email: hauke.lang@unimedizin-mainz.de. \\ Comment on: Hasselgren K, Isaksson B, Ardnor B, et al. Liver resection is beneficial for patients with colorectal liver metastases and extrahepatic \\ disease. Ann Transl Med 2020;8:109.
}

Submitted Jun 08, 2020. Accepted for publication Jun 21, 2020.

doi: $10.21037 /$ atm-20-4416

View this article at: http://dx.doi.org/10.21037/atm-20-4416

Colorectal cancer (CRC) is the third most frequent cancer worldwide. Distant metastases occur frequently. Nearly half of the patients present with or subsequently develop liver metastases, and about one third of patients have extrahepatic metastases already at the time of diagnosis or at a later date in the course of disease (1). Hepatic resection is the standard of care for colorectal liver metastases (CRLM) but only about $15-30 \%$ of patients are eligible for upfront surgery (2). Often, extensive or multifocal intrahepatic spread or the presence of extrahepatic disease (EHD) prevent resection for technical/functional reasons or make it questionable from an oncologic point of view.

In recent years there have been substantial developments in the treatment CRLM (2-7). The most significant changes were the invention of preoperative liver volume modulation techniques, the acceptance of parenchymal sparing resections as oncological adequate procedures and the introduction of effective chemotherapy regimens (2-5). With ongoing progress, the spectrum of indications for surgery for CRLM was steadily widened, and the presence of EHD was no longer considered a contraindication to liver resection provided that there was a chance of complete resection at all sites (8-10). Reports suggested a potential benefit and the possibility of achieving long-term survival and even cure (Table 1) (8-21). However, the literature is difficult to interpret due to a wide variability in selection criteria and in oncological pretreatment. Therefore, conclusions have to be drawn with caution, and the prognostic benefit after resection of liver and concomitant extrahepatic metastases is not yet clearly determined.
In a previous issue of Ann Transl Med, Hasselgren et al. reported in a retrospective study on outcome after hepatic resection for CRLM in the presence of EHD based on the data of the national Swedish liver registry (SweLiv) (22). Between 2009 and 2015, a total of 2,174 patients underwent surgical resection or intervention for CRLM, with 277 patients having concomitant EHD. In 246 cases metastases were confined to a single extrahepatic site. EHD was located in the lung in 179 patients, in lymph nodes in 69 patients, in the peritoneum in 29 patients and in other sites in 11 patients. Treatment of CRLM was either by hepatic resection in 244 patients or by ablation in 33 patients. The median overall survival of all 277 patients was 40 months, with 45 months after resection compared to 26 months after ablation of CRLM ( $\mathrm{P}=0.001)$. Single site EHD was associated with a median survival of 48 versus 35 months in EHD at two or three sites. Within the resection group, patients with concomitant lung metastases had a superior median survival (50 months) than with lymphatic (32 months) or peritoneal disease (28 months) $(\mathrm{P}=0.022$ and $\mathrm{P}=0.012$, respectively). Besides, patients who responded to preoperative chemotherapy had a much better median survival (48 months) compared to those with progressive disease under chemotherapy (13 months) upon multivariate analysis $(\mathrm{P}=0.004)$.

This is by far the largest report on surgical therapy of CRLM in the presence of EHD. It focusses on extrahepatic metastases in the lung, lymph nodes and the peritoneum. This stands to reason as these three sites account for about 
Table 1 Survival after resection of CRLM in the presence of EHD—review of literature

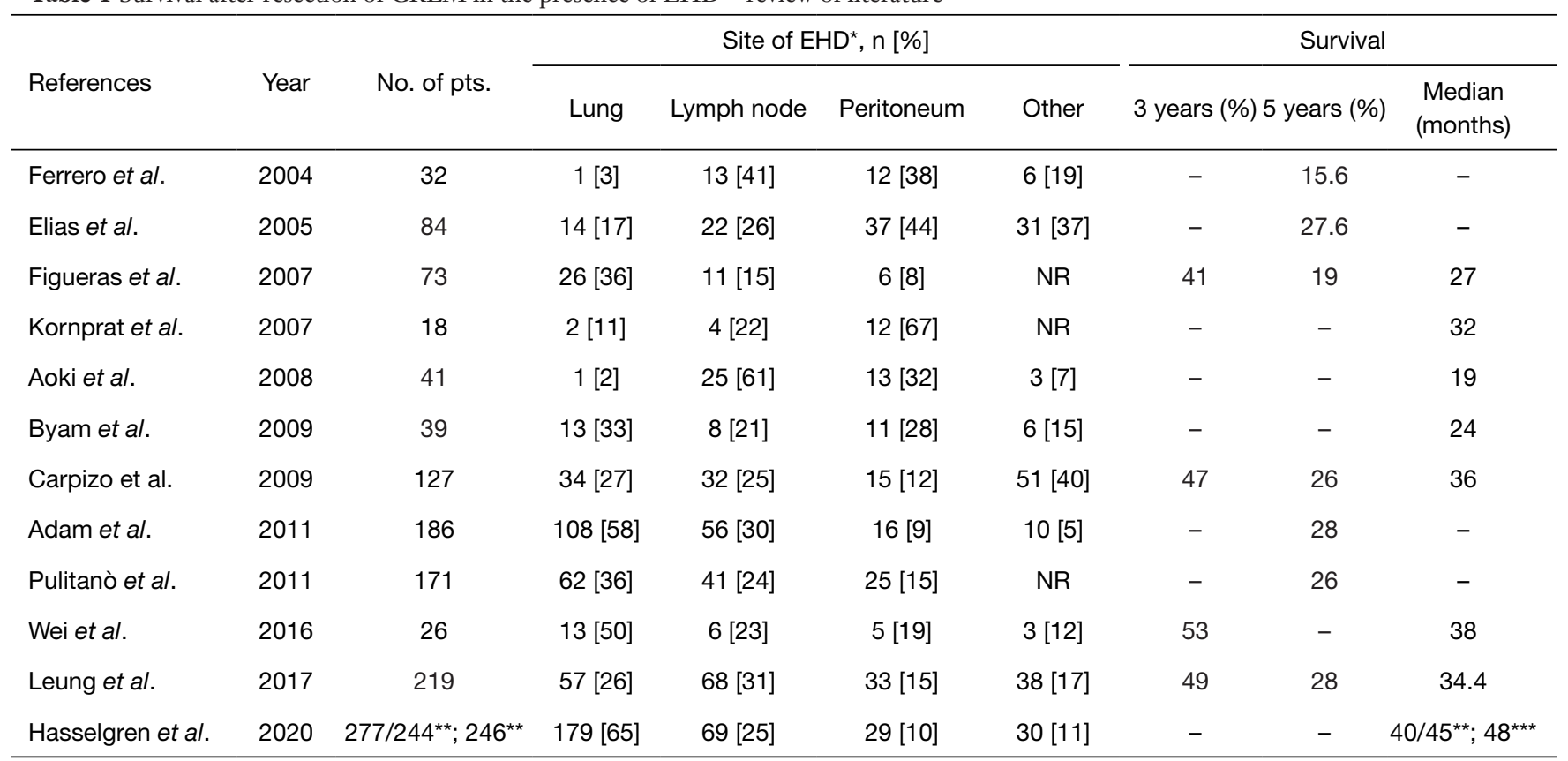

Review of literature 2004-2020 (not complete). ${ }^{*}$, total may be $\neq 100 \%$ due to multiple site EHD; ${ }^{* *}$, CRLM resected; ${ }^{* * *}$, single site EHD only disease. EHD, extrahepatic disease; NR, not reported; CRLM, colorectal liver metastases.

$80 \%$ or more EHD in patients considered for resection of CRLM (see Table 1).

The paper is of great importance in several respects. First, it is a large nationwide study including almost all patients undergoing surgical or interventional treatment for CRLM and EHD in Sweden. SweLiv is a validated national quality registry with a coverage of more than $95 \%$ of all liver resection and ablation cases for primary or secondary liver tumors. This gives a representative overview on the incidence of EHD in all resection candidates. Thus, it does not only reduce the influence of center specific inclusion criteria but reflects the nationwide daily surgical reality. Second, the short inclusion period of only 7 years supports a homogeneous group ensuring the comparability of staging procedures and systemic chemotherapy. Third, there is a comparably long median follow-up time after liver surgery of 26 months with $50 \%$ of patients being alive at the end of follow-up period. This allows to calculate reliable survival curves with high numbers at risk at least for the first 2 years.

The paper has two main messages:

(I) Patients benefit from resection of CRLM even in the presence of EHD.

(II) Patients whose tumors respond well to preoperative chemotherapy have a better prognosis than those with progressive disease.
This information strongly supports an increased consideration of resection for CRLM in the presence of EHD. And it stresses the importance of presurgical chemotherapy. Beyond its therapeutical effect response to systemic treatment is a common strategy to select patients for resection, in particular in systemic disease. These statements are of high clinical relevance. But, and not at all surprisingly the paper poses more questions than it answers. In particular it fails to more clearly show which patients are the best candidates for surgery, which benefit more and which less or not at all. Nowadays, even extended resections for CRLM can be performed with peri-operative mortality rates below $2 \%$ in most specialized hepatobiliary centers. Five-year survival rates range between $35-50 \%$ or even higher depending on patient selection $(4-7,23)$. In contrast, in metastatic CRC not amenable to surgery survival of 16-24 months can be achieved by chemotherapy and up to 30 months in highly selected patients $(24,25)$. These are the hallmarks a surgical approach to CRLM and EHD has to be measured against.

The Swedish paper reports on a median survival of 45 months (and of 48 months in single site EHD) which exceeds the best outcomes in patients receiving systemic treatment alone. The 5 -year survival rate of around $40 \%$ is almost in the range of what we can expect in CRLM without 
Table 2 Review of literature 2011-2020 (not complete), papers stratifying according to site of extrahepatic disease (lung, lymph nodes, peritoneum)

\begin{tabular}{|c|c|c|c|c|c|c|}
\hline References & Year & \multicolumn{2}{|c|}{ EHD } & \multicolumn{3}{|c|}{ Survival } \\
\hline \multirow[t]{3}{*}{ Adam et al. } & 2011 & Lung & 108 & 37 & 33 & 28 \\
\hline & & Lymph node & 56 & - & 20 & - \\
\hline & & Peritoneum & 26 & - & 0 & - \\
\hline \multirow{2}{*}{ Pulitanò et al. } & & Lymph node & $41^{*}$ & 43 & 27 & 29 \\
\hline & & Peritoneum & 25 & 32 & 26 & 32 \\
\hline \multirow[t]{2}{*}{ Hwang et al. } & 2014 & Lung & 852 & - & - & 45 \\
\hline & & Lymph node & 485 & - & - & 26 \\
\hline \multirow{2}{*}{ Hadden et al. } & & Lymph node & 559 & 35 & 15 & 24 \\
\hline & & Peritoneum & 378 & 37 & 17 & 29 \\
\hline \multirow[t]{3}{*}{ Leung et al. } & 2017 & Lung & 57 & 68 & 32 & 54 \\
\hline & & Lymph node & 68 & 32 & 14 & 24 \\
\hline & & Peritoneum & 33 & 54 & 42 & 40 \\
\hline \multirow[t]{3}{*}{ Hasselgren et al. } & 2020 & Lung & 179 & - & - & 50 \\
\hline & & Lymph node & 69 & - & - & 32 \\
\hline & & Peritoneum & 29 & - & - & 28 \\
\hline
\end{tabular}

${ }^{*}$, only hepatic pedicle lymph nodes. EHD, extrahepatic disease.

EHD. These results compare favorably to most reports in the literature. The reasons for this may be manifold but not least because of differences in the study periods. The paper by Hasselgren et al. refers exclusively to patients treated in the near past where there were significant improvements in the diagnostic work-up and availability of effective chemoand targeted therapy.

In addition, Hasselgren et al. describe a significantly longer survival after resection of CRLM with EHD in the lung than with in lymph nodes and peritoneum. This is in accordance to data of the literature suggesting that the site of extrahepatic metastases is a prognostic factor (Table 2) $(10,11,18,19,22,26)$.

To better assess the value of resection in CRLM and concomitant EHD and to further approach this topic scientifically a precise and comprehensive characterization of the entire malignant disease is indispensable. This includes detailed information about the location (right-sided versus left-sided) and stage of the primary tumor as well as an exact description of the number, size and distribution of all metastatic sites and the oncological pretreatment.

Clearly, complete removal of all intra- and extrahepatic tumor is essential to achieve long-term survival or even cure. Most data in this regard exist for patients having resectable liver and lung metastases (see Table 2). Although further evidence is lacking this setting is an accepted indication for surgery. In a systemic review of the literature enrolling 52 studies worldwide outcome was analysed in a total of 1,936 cases of R0-resections of CRLM and concomitant EHD (26). This meta-analyses revealed a median and 3- and 5-year survival after resection of liver and lung metastases ( 23 studies with $\mathrm{n}=574$ patients) of 42 months and $58 \%$ and $26 \%$, respectively, after resection of liver and peritoneal disease (16 studies with 378 patients) of 29 months and $37 \%$ and $17 \%$, and after resection of liver and lymph node metastases of 25 months and $35 \%$ and $15 \%$ 
(21 studies with 559 patients). Finally, resection of CRLM and EHD at multiple sites was associated with a median survival of 17 months and of $26 \%$ and $14 \%$ at 3 and 5 years, respectively (26).

These data are based on not at all homogeneous and comparable studies, each with its own patients' selection protocol and considerable differences in time period and systemic treatment. As such, the reported results vary widely from one study to another, e.g., the 5-year survival after resection of liver and lung metastases reaches up to $32 \%$ and after CRLM and peritoneal spread even up to $42 \%$ in one single center experience (21).

Similarly, in the Swedish study the heterogeneity of groups concerning the degree of EHD does not permit further conclusions. For instance, there is no differentiation between solitary or multiple lung metastases with regard to the extent of peritoneal disease. To better approach this setting, an exact number and size of lung metastases or an approximate quantification of peritoneal involvement (PI = peritoneal carcinomatosis index) would be of great relevance (27).

The results of resection of CRLM with nodal involvement are even more difficult to interpret. A precise anatomical mapping of removed lymph nodes is lacking. This is problematic as there may be differences in prognostic relevance of involved lymph nodes. While lymph node metastases in the hepatic hilum are regarded as locoregional spread of CRLM metastases in paraaortic or other lymph nodes are considered as distant tumor. This has been addressed by some authors reporting a 5 -year survival of $25 \%$ when there were metastases in lymph nodes at the hepatic hilum as opposed to $0 \%$ when paraaortic nodes were involved (28). However, data are limited, heterogeneous and sometimes even contradictory, so there is still uncertainty whether the site of lymph node metastases has a significant influence on prognosis (18,28-30).

In general, despite radical surgery the chance for cure remains low. This applies to all sites of EHD. Reported recurrence rates, even after resection of lung metastases, are extremely high up to more than $90 \%$ (21). This leads to the next question whether resection of CRLM is indicated in the presence of irresectable EHD. Again, most data exist for CRLM and concomitant pulmonary metastases. Maithel et al. followed patients after resection of liver metastases in the presence of irresectable lung metastases and revealed that survival was hardly impaired even in the case of progressive lung disease (31). Similarly, Mise et al. analysed the course of patients suffering from simultaneous liver and lung metastases. Outcome after complete removal of all metastatic sites was superior to resection of liver metastases only (32). Metastases treated with chemotherapy alone were associated with the worst prognosis. These findings suggest that the prognosis seems to be determined by the progression of hepatic tumor rather than by the pulmonary metastatic spread. This may justify the removal of CRLM but leaving lung metastases unresected. On the contrary, resection of CRLM in the presence of irresectable lymph node metastases or untreated peritoneal carcinomatosis is uncommon. The Swedish data suggests there is at least some benefit if CRLM can be removed completely. Further studies, i.e., comparing similar CRLM and EHD disease treated with resection of all metastases versus resection of CRLM only plus systemic treatment, are warranted to shed light on this frequent clinical scenario.

The Swedish authors neither differentiate between resected and non-resected EHD nor do they report on recurrence rates. There is even no histological proof of malignancy in all cases, in particular so in suspected pulmonary nodules and those with non-resected EHD. This may hamper the interpretation of their data. At the same time, this weak point can also be seen as a major strength of the paper as it reflects our daily reality. In clinical routine we frequently encounter CRLM concomitant with several pulmonary nodules where we do not know whether it is metastatic disease or not. The Swedish data support that we do not make a mistake when we go for liver resection in these cases. Similar to that, it is reasonable to proceed with resection if unexpected small-volume EHD, e.g., at the peritoneum or in hilar lymph nodes, is discovered during surgery for CRLM.

So far, reports have focused on CRLM with concomitant single site EHD mainly, but with increased knowledge also resection of liver metastases in the presence of multiple site EHD should be subject of further studies. The same applies for rare sites of EHD, such as metastases in the spleen, adrenal gland or ovaries which can be easily removed. The few data available in literature suggest results in the range of those achieved for resection of CRLM and lung metastases $(21,26)$. With further progress in neurosurgery and in stereotactic body radiation therapy (SBRT), even concomitant metastases to the brain need not inevitably be regarded as absolute contraindication to resection of CRLM.

A few clinical scores mainly based on the pathological characteristics of the CRLM, the EHD and response to chemotherapy proved to be helpful to predict outcome after 
surgery $(9,10,21)$. However, stratification on the basis of traditional markers often results in heterogeneous groups, and by doing so, subgroups that would benefit from a specific treatment are likely not to be detected.

In recent years, enhanced molecular profiling, mainly aiming at mutations of TP53, of the RAS/RAF pathway and of the members of the SMAD family, respectively, helped to better stratify patients for surgery of CRLM (33-35). In particular, KRAS mutations have been shown to be associated with a worse prognosis after resection of CRLM and a higher risk of overall and lung-specific recurrence. Interestingly, in a preliminary report resection of single site colorectal metastases in the lung or peritoneum was not associated with an impaired survival in the presence of KRAS or NRAS mutations (36). Further studies are warranted to examine this topic more closely and to better understand these findings. It is most likely that a more detailed knowledge of the molecular pattern of both liver and extrahepatic metastases will help to identify factors influencing the outcome after surgery in this setting and will sharpen our selection criteria.

In summary, the presented work encountered in a large, homogeneous nationwide population is of high clinical relevance as it supports that liver resection for CRLM is beneficial even in the presence of EHD.

"Biology is King, selection of cases is Queen, and the technical details of surgical procedures are the Princes and Princesses ..." (37). This we need to have in mind when trying to define the role of resection of CRLM in the presence of EHD. While surgical procedures are on the way to be well defined, there is a lot a lot of work ahead to increase knowledge of biology and to improve patients' selection. The Swedish data both justify and stimulate further efforts in this field.

\section{Acknowledgments}

Funding: None.

\section{Footnote}

Provenance and Peer Review: This article was commissioned by the editorial office, Annals of Translational Medicine. The article did not undergo external peer review.

Conflicts of Interest: The author has completed the ICMJE uniform disclosure form (available at http://dx.doi. org/10.21037/atm-20-4416). The author has no conflicts of interest to declare.
Ethical Statement: The author is accountable for all aspects of the work in ensuring that questions related to the accuracy or integrity of any part of the work are appropriately investigated and resolved.

Open Access Statement: This is an Open Access article distributed in accordance with the Creative Commons Attribution-NonCommercial-NoDerivs 4.0 International License (CC BY-NC-ND 4.0), which permits the noncommercial replication and distribution of the article with the strict proviso that no changes or edits are made and the original work is properly cited (including links to both the formal publication through the relevant DOI and the license). See: https://creativecommons.org/licenses/by-nc-nd/4.0/.

\section{References}

1. Manfredi S, Lepage C, Hatem C, et al. Epidemiology and management of liver metastases from colorectal cancer. Ann Surg 2006;244:254-9.

2. Bismuth H, Adam R, Levi F, et al. Resection of nonresectable liver metastases from colorectal cancer after neoadjuvant chemotherapy. Ann Surg 1996;224:509-20.

3. Jaeck D, Oussoultzoglou E, Rosso E, et al. A two-stage hepatectomy procedure combined with portal vein embolization to achieve curative resection for initially unresectable multiple and bilobar colorectal liver metastases. Ann Surg 2004;240:1037-49.

4. Torzilli G, Vigano L, Cimino M, et al. Is enhanced onestage hepatectomy a safe and feasible alternative to the two-stage hepatectomy in the setting of multiple bilobar colorectal liver metastases? A comparative analysis between two pioneering centers. Dig Surg 2018;35:323-32.

5. Adam R, Wicherts DA, de Haas RJ, et al. Patients with initially unresectable colorectal liver metastases: is there a possibility of cure? J Clin Oncol 2009;27:1829-35.

6. Baumgart J, Jungmann F, Bartsch F, et al. Two-stage hepatectomy and ALPPS for advanced bilateral liver metastases: a tailored approach balancing risk and outcome. J Gastrointest Surg 2019;23;2391-400..

7. Lang H. ALPPS for colorectal liver metastases. J Gastrointest Surg 2017;21:190-2.

8. Carpizo DR, D'Angelica M. Liver resection for metastatic colorectal cancer in the presence of extrahepatic disease. Lancet Oncol 2009;10:801-9.

9. Imai K, Benitez CC, Allard MA, et al. Potential of a cure in patients with colorectal liver metastases and concomitant extrahepatic disease. J Surg Oncol 2017;115:488-96. 
10. Adam R, de Haas RJ, Wicherts DA, et al. Concomitant extrahepatic disease in patients with colorectal liver metastases: when is there a place for surgery? Ann Surg 2011;253:349-59.

11. Ferrero A, Polastri R, Muratore A, et al. Extensive resections for colorectal liver metastases. J Hepatobiliary Pancreat Surg 2004;11:92-6.

12. Elias D, Liberle G, Vernerey D, et al. Hepatic and extrahepatic colorectal metastases: when resectable, their localization does not matter, but their total number has a prognostic effect. Ann Surg Oncol 2005;12:900-9.

13. Figueras J, Torras J, Valls C, et al. Surgical resection of colorectal liver metastases in patients with expanded indications: a single-center experience with 501 patients. Dis Colon Rectum 2007;50:478-88.

14. Kornprat P, Jarnagin WR, Gonen M, et al. Outcome after hepatectomy for multiple (four or more) colorectal metastases in the era of effective chemotherapy. Ann Surg Oncol 2007;14:1151-60.

15. Aoki T, Umekita N, Tanaka S, et al. Prognostic value of concomitant resection of extrahepatic disease in patients with liver metastases of colorectal origin. Surgery 2008;143:706-14.

16. Byam J, Reuter NP, Woodall CE, et al. Should hepatic metastatic colorectal cancer patients with extrahepatic disease undergo liver resection/ablation? Ann Surg Oncol 2009;16:3064-9.

17. Carpizo DR, Are C, Jarnagin W, et al. Liver resection for metastatic colorectal cancer in the patients with concurrent extrahepatic disease. Results in 127 patients treated at a single center. Ann Surg Oncol 2009;16:2138-46.

18. Pulitanò C, Bodinghauer M, Aldrighetti L, et al. Liver resection for colorectal metastases in presence of extrahepatic disease: results from an international multiinstitutional analysis. Ann Surg Oncol 2011;18:1380-8.

19. Hwang M, Jayakrishnan TT, Green DE, et al. Systematic review of outcomes of patients undergoing resection for colorectal liver metastases in the setting of extrahepatic disease. Eur J Cancer 2014;50:1747-57.

20. Wei AC, Coburn NG, Devitt KS, et al. Survival following resection of intra- and extra-hepatic metastases from colorectal cancer: A phase II trial. Ann Surg Oncol 2016;23:2644-51.

21. Leung U, Gönen M, Allen PJ, et al. Colorectal cancer liver metastases and concurrent extrahepatic disease treated with resection. Ann Surg 2017;265:158-65.

22. Hasselgren $\mathrm{K}$, Isaksson $\mathrm{B}$, Ardnor $\mathrm{B}$, et al. Liver resection is beneficial for patients with colorectal liver metastases and extrahepatic disease. Ann Transl Med 2020;8:109.

23. Brouquet A, Abdalla EK, Kopetz S, et al. High survival rate after two-stage resection of advanced colorectal liver metastases:response-based selection and complete resection define outcome. J Clin Oncol 2011;10:1083-90.

24. Heinemann V, von Weikersthal LF, Decker T, et al. FOLFIRI plus cetuximab versus FOLFIRI plus bevacizumab as first-line treatment for patients with metastatic colorectal cancer (FIRE-3). A randomized open-label, phase 3 trial. Lancet Oncol 2014;15:1065-75.

25. Venook AP, Niedzwiecki D, Lenz HJ, et al. Effect of first-line chemotherapy combined with Cetuximab or Bevacizumab on overall survival in patients with KRAS wild-typ advanced or metastatic colorectal cancer. JAMA 2017;317:2392-401.

26. Hadden WJ, de Reuver PR, Brown K, et al. Resection of colorectal liver metastases and extra-hepatic disease: a systematic review and proportional meta-analysis of survival outcomes. HPB (Oxford) 2016;18:209-20.

27. Elias D, Benizri E, Pocard M, et al. Treatment of synchronous peritoneal carcinomatosis and liver metastases from colorectal cancer. Eur J Surg Oncol 2006;32:632-36.

28. Adam R, de Haas RJ, Wicherts DA, et al. Is hepatic resection justified after chemotherapy in patients with colorectal liver metastases and lymph node involvement? J Clin Oncol 2008;26:3672-80.

29. Okuno M, Goumard C, Mizuno T, et al. Prognostic impact of perihepatic lymph node metastases in patients with resectable colorectal liver metastases. Br J Surg 2018;105:1200-9.

30. Pulitanò C, Bodingbauer M, Aldrighetti L, et al. Colorectal liver metastases in the setting of lymph node metastasis:defining the benefit of surgical resection. Ann Surg Oncol 2012;19:435-42.

31. Maithel SK, Ginsberg MS, D'Amico F, et al. Natural history of patients with subcentimeter pulmonary nodules undergoing hepatic resection for metastatic colorectal cancer. J Am Coll Surg 2010;210:31-8.

32. Mise Y, Kopetz S, Mehran RJ, et al. Is complete liver resection without resection of synchronous lung metastases justified? Ann Surg Oncol 2015;22:1585-92.

33. Vauthey JN, Kawaguchi Y. Innovation and future perspectives in the treatment of colorectal liver metastases. J Gastrointest Surg 2020;24:492-6.

34. Kawaguchi Y, Kopetz S, Newhook TE, et al. Mutation Status of RAS, TP53, and SMAD4 is superior to mutation status of RAS alone for predicting prognosis after resection of colorectal liver metastases. Clin Cancer Res 
2019;25:5843-51.

35. Lang H, Baumgart J, Heinrich S, et al. Extended molecular profiling improves stratification and prediction of survival after resection of colorectal liver metastases. Ann Surg 2019;270:799-805.

36. Passot G, Kim BJ, Glehen O, et al. Impact of mutations

Cite this article as: Lang H. Liver resection is beneficial for patients with colorectal liver metastases and extrahepatic disease. Ann Transl Med 2020;8(18):1122. doi: 10.21037/atm20-4416 in metastatic colorectal cancer after potentially curative resection. Does site of metastases matter? Ann Surg Oncol 2018;25:179-87.

37. Cady B. Basic Principles in Surgical Oncology. Arch Surg 1997;132:338-46. 